\title{
Can the Identification of an Idle Line Facilitate Its Removal? A Comparison Between a Proposed Guideline and Clinical Practice
}

\author{
Areeba Kara, MD, MS, FACP ${ }^{1 \star}$, Cynthia S. Johnson, MA², Michelle Murray, MD , Jill Dillon, RN, MSN4, Siu L. Hui, PhD ${ }^{5}$
}

${ }^{1}$ Inpatient Medicine, Indiana University Health Physicians, and Indiana University School of Medicine, Indianapolis, Indiana; ${ }^{2}$ Indiana University School of Medicine and Department of Biostatistics, Richard M. Fairbanks School of Public Health, Indianapolis, Indiana; ${ }^{3}$ Internal Medicine-Pediatrics residency, Indiana University School of Medicine, Indianapolis, Indiana; ${ }^{4}$ Methodist Hospital, Indiana University Health, Indianapolis, Indiana; ${ }^{5}$ Regenstrief Institute, Indianapolis, Indiana.

BACKGROUND: There are 250,000 cases of central lineassociated blood stream infections in the United States annually, some of which may be prevented by the removal of lines that are no longer needed.

OBJECTIVE: To test the performance of criteria to identify an idle line as a guideline to facilitate its removal.

METHODS: Patients with central lines on the wards were identified. Criteria for justified use were defined. If none were met, the line was considered "idle." We proposed the guideline that a line may be removed the day following the first idle day and compared actual practice with our proposed guideline.

RESULTS: One hundred twenty-six lines in 126 patients were observed. Eighty-three (65.9\%) were peripherally inserted central catheters. Twenty-seven percent $(n=34)$ were placed for antibiotics. Seventy-six patients had lines removed prior to discharge. In these patients, the line was in place for 522 days, of which $32.7 \%$ were idle. The most common reasons to justify the line included parenteral antibiotics and meeting systemic inflammatory response (SIRS) criteria. In 11 (14.5\%) patients, the line was removed prior to the proposed guideline. Most $(n=36,47.4 \%)$ line removals were observed to be in accordance with our guideline. In another 29 (38.2\%), line removal was delayed compared to our guideline.

CONCLUSIONS: Idle days are common. Central line days may be reduced by the consistent daily reevaluation of a line's justification using defined criteria. The practice of routine central line placement for prolonged antibiotics and the inclusion of SIRS criteria to justify the line may need to be reevaluated. Journal of Hospital Medicine 2016;11:489-493. (C) 2016 Society of Hospital Medicine
Infections acquired in the hospital are termed healthcare-associated infections (HAIs) and include central line-associated blood stream infections (CLABSIs). Among HAIs, CLABSIs cause the highest number of preventable deaths. ${ }^{1}$ Central venous catheters (CVCs) or central lines are commonly used in the hospital. ${ }^{2}$ Each year their use is linked to 250,000 cases of CLABSIs in the United States. ${ }^{3}$ Some CLABSIs may be prevented by the prompt removal of the line. ${ }^{4}$ However, CVCs are often retained after their clinical indication has lapsed and are then referred to as "idle" lines. ${ }^{5,6}$ In this work, we propose and theoretically test a guideline to facilitate the safe removal of an idle line by observing the agreement and disagreement between actual practice and the proposed guideline.

\footnotetext{
*Address for correspondence and reprint requests: Areeba Kara, MD, Inpatient Medicine, Indiana University Health Physicians, Indiana University School of Medicine, Noyes Pavilion Suite 640, 1701 N Senate Avenue, Indianapolis, IN 46202-1239; Telephone: 317-962-2894; Fax number 317-963-5285; E-mail: akara@iuhealth.org

Additional Supporting Information may be found in the online version of this article.

Received: November 9, 2015; Revised: January 31, 2016; Accepted: February 2, 2016

2016 Society of Hospital Medicine DOI 10.1002/jhm.2573

Published online in Wiley Online Library (Wileyonlinelibrary.com).
}

\section{METHODS \\ Setting}

This work was conducted at a large, urban, tertiary care, academic health center in the United States as a collaborative effort to improve quality at our institution. ${ }^{7}$

\section{Design and Patients}

The reports linked with the electronic medical records at our institution include a daily, ward-by-ward listing of patients who have access other than a peripheral line in place. This "central line dashboard" accesses the information on intravenous access charted by bedside nurses to create a list of patients on every ward who have any kind of central access. Temporary central venous lines (CVLs), peripherally inserted central catheters (PICCs), ports, and dialysis catheters are all included. The unit charge nurses and managers use this dashboard to facilitate compliance with line care bundles. We used this source to identify patients with either type of CVC (CVLs or PICCs) on 8 days in August 2014, September 2014, and October 2014. Patients were included if they had a CVC and were on a general medical or surgical ward bed on audit day. CVLs at all sites were included (femoral, subclavian, and internal jugular). Patients in an intensive care unit (ICU) or progressive care unit on the day of the audit were excluded. 


TABLE 1. Criteria to Justify the Presence of a
Central Line
IV access needs
Expected duration of IV antibiotics $>6$ days
Administration of TPN
Anticipated requirement of home IV medications
Requirement of IV medications with documented difficult access
Hemorrhage requiring blood transfusions
Requiring more than 3 infusions
Requiring more than 2 infusions and blood transfusions
Abnormal vitals
Diastolic blood pressure $>120 \mathrm{~mm}$ Hg
Systolic blood pressure $<90 \mathrm{~mm} \mathrm{Hg}$
Systolic blood pressure $>200 \mathrm{~mm}$ Hg
Heart rate $>120$ beats per minute
Heart rate $<50$ beats per minute
Respiratory rate $>30$ breaths per minute
Respiratory rate $<10$ breaths per minute
Oxygen saturation $<90 \%$ as measured by pulse oximetry
Meeting SIRS criteria ( 2 or more of the following present)
Temp $>38^{\circ} \mathrm{C}$, Temp $<36^{\circ} \mathrm{C}$, heart rate $>90$ beats per minute, respiratory rate $>20$ breaths
per minute, WBC $>12,000 / \mathrm{mm}^{3}$, WBC $<1,000 / \mathrm{mm}^{3}$, bandemia $>10 \%$

NOTE: If none of these criteria were met, the line was considered idle for that day. Abbreviations: IV, intravenous; TPN, total parenteral nutrition; SIRS, systemic inflammatory response syndrome; WBC, white blood count.

Patients whose catheters were for chemotherapy and those admitted for a transplant or receiving palliative or hospice care were also excluded.

\section{Data Collection}

A protocol for data collection was written out, and a training session was held to review definitions, data sources, and methods to ensure consistency. Two authors (M.M. and J.D.) assisted by an experienced clinical nurse specialist collected data on the patients captured on audit days. Each chart was reviewed on the day of the audit, the 2 days preceding the audit day, and then followed until the patient was either discharged from the hospital or transferred to a higher level of care, died, or transitioned to palliative or hospice care. Demographics, details about the line, and the criteria for justified use were extracted from the electronic medical record.

\section{Definitions}

\section{Justified and Idle Days}

To justify the presence of a CVC on any given day, we used criteria that fell under 3 categories: intravenous (IV) access needs, unstable vitals, or meeting sepsis/systemic inflammatory response syndrome (SIRS) criteria (Table 1). For vital signs, a single abnormal reading was counted as fulfilling criteria for that day. If no criterion for justified use was met, the line was considered idle for that day.

Qualifying IV access needs were defined similarly to those previously used, ${ }^{5,6}$ whereas those for SIRS followed the current consensus. ${ }^{8}$ To determine the number of IV medications or infusions, the medication administration record was reviewed. If 3 or more infusions were found, their compatibility was checked using the same database that nurses use at our institution. Difficult IV access was inferred from the indication for line placement, coupled with the absence of documentation of a peripheral IV. Clinical progress notes were reviewed to extract information on the length of proposed IV antibiotic courses, and discharge instructions were reviewed to verify whether the line was removed prior to discharge or not. The cutoffs for diastolic blood pressure, respiratory rate, and oxygen saturation used to label patients hemodynamically labile are the same as those used by previous authors and also constitute the definition of "hypertensive urgency." ",9 However, we diverged from the values previously used for tachycardia, bradycardia, and systolic hypotension using heart rates $>120$ and $<50$ beats per minute (compared to $>130$ and $<40$ beats per minute) and systolics $<90 \mathrm{~mm} \mathrm{Hg}$ (compared to $<80$ $\mathrm{mm} \mathrm{Hg}$ ) to justify the line. ${ }^{5}$ Early warning scores have been used to identify hospitalized ward patients who are at risk for clinical deterioration. Although each score utilizes different thresholds, the risk for clinical deterioration increases as the vitals worsen. ${ }^{10}$ Bearing this in mind, the thresholds we elected to use are more clinically conservative and also parallel the nursing call orders currently used at our institution.

\section{Proposed Guideline}

We propose the guideline that a CVC may be safely removed the day after the first idle day.

\section{RESULTS}

A total of 126 lines were observed in 126 patients. Eighty-three $(65.9 \%)$ of the lines were PICCs. The remaining $43(34.1 \%)$ were CVLs. The indications for line placement were distributed between the need for central access, total parenteral nutrition, or antibiotics (Table 2).

\begin{tabular}{lc}
\hline \multicolumn{2}{l}{ TABLE 2. Description of the Study Cohort } \\
\hline Description & Value \\
\hline Age in yrs mean (SD) & $55.7(18)$ \\
Gender, $n$ (\%) & \\
$\quad$ Female & $66(52.4)$ \\
$\quad$ Male & $60(47.6)$ \\
Type of line, $n$ (\%) & \\
PICC & $83(65.9)$ \\
CVL & $43(34.1)$ \\
Indication for line placement, $n$ (\%) & \\
Meds requiring central access or TPN & $36(28.6)$ \\
Antibiotics & $34(27.0)$ \\
Hemodynamic instability & $30(23.8)$ \\
Poor access with multiple IV medications & $18(14.3)$ \\
Unknown & $8(6.3)$ \\
Line removed prior to discharge, $n$ (\%) & $76(60.3)$ \\
Yes & $50(39.7)$ \\
$\quad$ No &
\end{tabular}

NOTE: Abbreviations: CVL, central venous line; IV, intravenous; PICC, peripherally inserted central catheter; $\mathrm{SD}$, standard deviation; TPN, total parenteral nutrition. 


\begin{tabular}{lcc} 
TABLE 3. Criteria Met for the 351 Justified Line \\
Days \\
$\begin{array}{l}\text { Criteria } \\
\text { N }\end{array}$ \\
\hline No. of factors justifying use & \\
\hline 1 & 184 & $52.4 \%$ \\
2 & 127 & $36.2 \%$ \\
$>2$ & 40 & 11.4 \\
Reason for justifying line* & & \\
Anticipate home or $>6$ days of antibiotic use & 181 & 51.6 \\
SIRS criteria & 124 & 35.3 \\
TPN & 96 & 27.4 \\
Hemodynamic instability based on hr and bp & 78 & 22.2 \\
Poor access with need for IV medications & 57 & 16.2 \\
Respiratory rate (<10 or $>30 /$ minute) & 25 & 7.1 \\
Active hemorrhage requiring transfusions & 12 & 3.4 \\
$>3$ infusions & 6 & 1.7 \\
\hline
\end{tabular}

NOTE: Abbreviations: IV, intravenous; SIRS, systemic inflammatory response syndrome; TPN, total parenteral nutrition; hr: heart rate; bp. blood pressure. * ${ }^{*}$ Totals exceed $100 \%$ because multiple indications may exist.

Out of the 126 patients, $50(39.7 \%)$ were discharged from the hospital, died, were transferred to a higher level of care, or transitioned to palliative or hospice care with the line in place. In the remaining 76 patients, the audit captured 635 days, out of which a line was in place for $522(82.2 \%)$ days. Of these 522 days, the line's presence was justified by our criteria for $351(67.2 \%)$ days. The most common reason for a line to be justified on any given day was the need for antibiotics followed by the presence of SIRS criteria (Table 3). The remaining 171 (32.7\%) days were idle.
A comparison of the actual removal of the 76 central lines in practice relative to the proposed guideline of removing it the day following the first idle day is displayed in Figure 1. The central line was removed prior to our proposed guideline in 11 (14.5\%) patients, and waiting for an idle day in these patients would have added 46 line days. In almost half the patients ( $\mathrm{n}=36,47.4 \%)$, the line was removed in agreement with the proposed guideline. None of the patients in whom the line was removed prior to or in accordance with our proposed guideline required a line reinsertion. Line removal was delayed in 29 $(38.2 \%)$ patients when compared to our proposed guideline. In these patients, following the guideline would have created 122 line-free days. Most ( $\mathrm{n}=$ $102,83.6 \%$ ) of these potential line-free days were idle. Twenty $(16.4 \%)$ were justified, of which half $(\mathrm{n}=10)$ were justified by meeting SIRS criteria.

\section{DISCUSSION}

Approximately 1 in every 25 inpatients in the United States has at least $1 \mathrm{HAI}$ on any given day. ${ }^{11}$ The case fatality rate from a CLABSI may be as high as $12 \%$, and up to $70 \%$ of these infections may be preventable. ${ }^{1,12}$ Interventions successful in decreasing CLABSIs have focused on patients in ICUs. ${ }^{13}$ However, CVCs are increasingly prevalent outside the ICU, with over 4.5 million line days in non-ICU beds reported to the National Healthcare Safety Network in 2012 compared to 2.5 million in $2010 .{ }^{2,14}$ However, adherence rates to infection control practices may be lower on

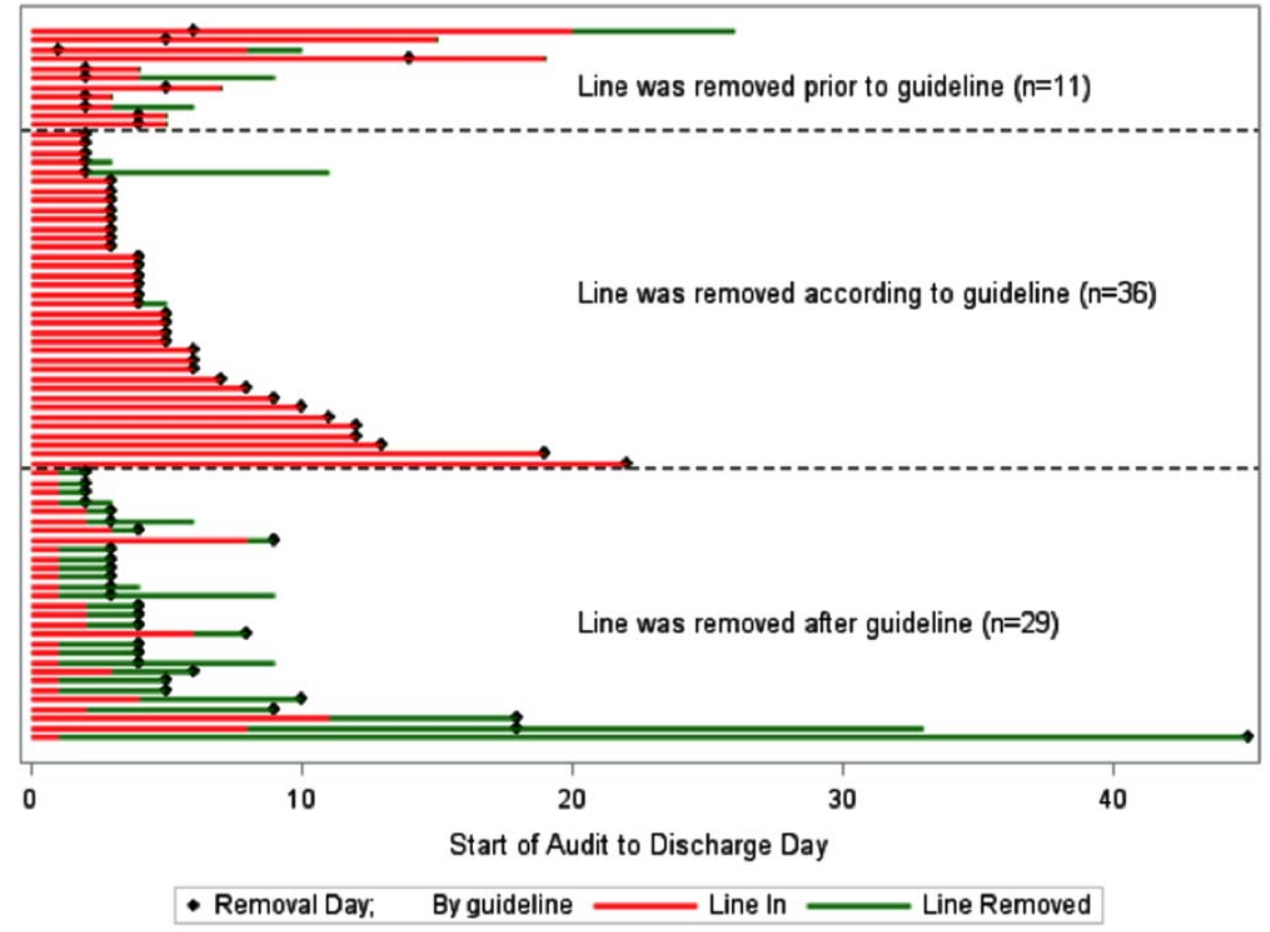

FIG. 1. Pictorial demonstration of the comparison between line removal in practice and the proposed guideline of removing it the day following the first idle day. Each bar represents 1 of the 76 patients in whom the line was removed prior to discharge. The diamond represents the actual removal of the line in practice. The bar is red to indicate that the line will remain in place according to our proposed guideline. It turns to green the day following the first idle day indicating that our guideline would recommend line removal. 
the wards than in the ICUs. ${ }^{6,15}$ Consequently, although the number of CLABSIs has declined over the last decade, most are now occurring outside the ICU. ${ }^{16}$ These trends underscore the need to develop strategies aimed at CLABSI prevention on the floors.

Analogous to the 'life cycle' of a urinary catheter described by Meddings et al., ${ }^{17}$ strategies to prevent CLABSIs and other CVC-related complications may be designed around the life cycle of a CVC. The life cycle starts with insertion and moves on to the maintenance, removal, and possible reinsertion of the line. The process thus starts with the decision to place the line. Over the last decade, this decision making has changed in part due to PICCs. This shift is reflected in PICC prevalence rates: in 2001, $11 \%$ of audited central lines were PICCs compared to $56 \%$ in $2007.5,6$ In our audit, $66 \%$ of the CVCs were PICCs. This increase in the use of PICCs may be attributable to the ease and safety of their placement coupled with the increased availability of vascular access placement teams. ${ }^{18}$ The risk of overuse that may result from such expediency may be countered by adhering to guidelines such as the Michigan Appropriateness Guide for Intravenous Catheters, which provides both clinically detailed guidance and an impetus for reflective decision making around intravenous access. ${ }^{19}$

The placement of CVCs for prolonged parenteral antibiotics may be a particular subset that bears further exploration. Similar to previous reports, we found that a large number of the CVCs were both inserted for and justified by the need for IV antibiotics. ${ }^{5}$ Guidelines delineated by the Infectious Diseases Society of America regarding outpatient parenteral antibiotics weigh both the duration of therapy and the antimicrobial's potential for causing phlebitis when recommending the type of intravascular access. ${ }^{20}$ Many courses may therefore be completed through peripheral or midline catheters. Developing strong partnerships between infectious disease specialists, hospitalists, and the facilities or home-care services treating these patients may curtail the use of CVCs for antimicrobial administration.

The main focus of our work is on facilitating the safe removal of CVCs. The risk of CLABSIs increases each day a CVC is in place, and guidelines to prevent CLABSIs include recommendations to promptly remove nonessential catheters. ${ }^{4,21}$ There is also an emerging understanding that the risk of a PICCrelated CLABSI approaches that from a traditional central line in hospitalized patients, and PICCs confer an increased risk of venous thromboembolism. ${ }^{18,22}$ Although nearly half of surveyed hospitalists recently reported leaving PICCs in place until discharge day, our data suggest that this practice may be driven by the trajectory of a patient's recovery as much as by knowledge gaps related to the use of PICCs. ${ }^{23}$ In nearly half the instances, clinical practice already mirrors our proposed guideline, with line removal coin- ciding with both the timing proposed by our guideline and discharge day. However, there is room for improvement, as line removal may have been expedited in the 29 patients in whom the line was retained after the first idle day. Maintaining an awareness of its presence and weighing its risks and benefits daily may facilitate the removal of a CVC. Based on the recent findings that up to a quarter of clinicians are unaware that their patients have a central line, the mere reminder of the presence of a line using such criteria may expedite its removal by triggering a purposeful reassessment of its ongoing need. ${ }^{24}$ Premature CVC removal requiring line reinsertion is an unintended consequence that may emerge from the earlier removal of lines. In our sample, none of the patients who had lines removed either prior to or in accordance with our proposed guideline required a line reinsertion. In addition to line reinsertion, delays in laboratory testing and reporting due to the unavailability of access, increased patient discomfort, or increased workload on the bedside nurse or vascular access team must also be considered when implementing strategies aimed at decreasing line days.

We envisage using these criteria to both empower practitioners with knowledge and foster shared accountability between all team members by using a uniform tool. This can occur through partnerships between infection control, clinical nurse specialists, bedside nursing, and physicians. The electronic medical record could be leveraged to scan the record for the criteria and create a notification when the line becomes idle. In alignment with the Michigan Appropriateness Guide for Intravenous Catheters guidelines, we do not support the removal of lines by nursing staff without physician notification. ${ }^{19}$ Such principles have been successfully harnessed in strategies to prevent both catheter-associated urinary tract infections and CLABSIs in ICUs. ${ }^{13,25}$ In light of the complexity surrounding the decision making for CVCs, our criteria were focused on the wards and erred on the side of clinical caution. This clinical conservatism is apparent in the patients in whom lines were removed prior to what our guideline would propose, yet none of the patients required a line reinsertion. As concerns about recrudescent clinical instability may drive decision making around line removal, such conservatism may be warranted initially. However, the fidelity of these criteria in the clinical setting will need prospective validation. In particular, the inclusion of SIRS criteria may have led to an overestimation of justified days. Further studies may be needed to refine the criteria and find a clinical hierarchy that balances the risks and benefits of retaining a central line.

Our work has certain limitations. It is a single center's experience, and our findings may not therefore be generalizable. Except for when the indication for the line was for difficult access, we did not attempt to verify the presence of a peripheral IV. This, in 
combination with the inclusion of SIRS criteria, likely leads to an underestimation of idle days. In the interest of focusing on patients in whom the decision making around a line would be the least controversial, we did not continue to follow patients who were transferred to a higher level of care. It is possible, however, that these transfers were precipitated by lineassociated complications such as sepsis and would be important to track. We did not measure the agreement between data collectors, although definitions and methodologies were standardized and reviewed prior to data collection. As this was an observational assessment of a proposed guideline, we cannot predict how the recommendations generated by it will be received by clinicians. Although this may prove to be a barrier in adoption, we hope that the conversation it initiates leads to change.

Hospitalists are positioned to potentially influence the entire life cycle of a central line on the floor. Strategies can be enacted at each stage to help decrease the potential of harm from these devices to our patients. Creating and testing criteria and guidelines such as we propose represents just 1 such strategy in a multidisciplinary effort to provide the best possible care we can.

\section{Acknowledgements}

The authors thank Jennifer Dunscomb, Kristen Kelly, and their teams, and Deanna Sidwell, Todd Biggerstaff, Joan Miller, Rob Clark, and the tireless providers at Indiana University Health Methodist Hospital for their support.

Disclosures: This work was supported by the Indiana University Health Values Grant for research. The authors have no conflicts of interests to report.

\section{References}

1. Umscheid CA, Mitchell MD, Doshi JA, Agarwal R, Williams K, Brennan PJ. Estimating the proportion of healthcare-associated infections that are reasonably preventable and the related mortality and costs. Infect Control Hosp Epidemiol. 2011;32(2):101-114.

2. Dudeck MA, Weiner LM, Allen-Bridson K, et al. National Healthcare Safety Network (NHSN) report, data summary for 2012, deviceassociated module. Am J Infect Control. 2013;41(12):1148-1166.

3. Maki DG, Kluger DM, Crinch CJ. The risk of bloodstream infection in adults with different intravascular devices: a systematic review of 200 published prospective studies. Mayo Clin Proc. 2006;81(9): 1159-1171.

4. O'Grady NP, Alexander M, Burns LA, et al. Guidelines for the prevention of intravascular catheter-related infections. Clin Infect Dis. 2011;52(9):e162-e193.

5. Chernetsky Tejedor S, Tong D, Stein J, et al. Temporary central venous catheter utilization patterns in a large tertiary care center: tracking the "idle central venous catheter." Infect Control Hosp Epidemiol. 2012;33(1):50-57.

6. Trick WE, Vernon M, Welbel SF, Wisniewski MF, Jernigan JA, Weinstein RA. Unnecessary use of central venous catheters: the need to look outside the intensive care unit. Infect Control Hosp Epidemiol. 2004;25(3):266-268.

7. IU Health Methodist Hospital website. Available at: http://iuhealth. org/methodist/aboIut. Accessed October 20, 2014.

8. Bone RC, Balk RA, Cerra FB, et al. Definitions for Sepsis and Organ Failure and Guidelines for the Use of Innovative Therapies in Sepsis. The ACCP/SCCM Consensus Conference Committee. American College of Chest Physicians/Society of Critical Care Medicine. Chest. 2009;136(5 suppl):e28.

9. Pak KJ, Hu T, Fee C, Wang R, Smith M, Bazzano LA. Acute hypertension: a systematic review and appraisal of guidelines. Ochsner J. 2014; 14(4):655-663.

10. Churpek MM, Yuen TC, Edelson DP. Predicting clinical deterioration in the hospital: the impact of outcome selection. Resuscitation. 2013; 84(5):564-568.

11. Magill SS, Edwards JR, Bamberg W, et al. Multistate point-prevalence survey of health care-associated infections. N Engl J Med. 2014; 370(13):1198-1208.

12. Klevens RM, Edwards JR, Richards CL, et al. Estimating health careassociated infections and deaths in U.S. hospitals, 2002. Public Health Rep. 2007;122(2):160-166.

13. Pronovost P, Needham D, Berenholtz S, et al. An intervention to decrease catheter-related bloodstream infections in the ICU. N Engl J Med. 2006;355(26):2725-2732.

14. Dudeck MA, Horan TC, Peterson KD, et al. Data summary for 2011, device-associated module. Centers for Disease Control and Prevention. National Healthcare Safety Network (NHSN) Report. Available at: http:// www.cdc.gov/nhsn/PDFs/dataStat/NHSN-Report-2011-Data-Summary. pdf. Published April 1, 2013. Last accessed January 2015.

15. Burdeu G, Currey J, Pilcher D. Idle central venous catheter-days pose infection risk for patients after discharge from intensive care. Am J Infect Control. 2014;42(4):453-455.

16. Liang SY, Marschall J. Update on emerging infections: news from the Centers for Disease Control and Prevention. Vital signs: central lineassociated blood stream infections-United States, 2001, 2008, and 2009. Ann Emerg Med. 2011;58(5):447-451.

17. Meddings J, Rogers MAM, Krein SL, Fakih MG, Olmsted RN, Saint S. Reducing unnecessary urinary catheter use and other strategies to prevent catheter-associated urinary tract infection: an integrative review. BMJ Qual Saf. 2014;23(4):277-289.

18. Chopra V, O'Horo JC, Rogers MAM, Maki DG, Safdar N. The risk of bloodstream infection associated with peripherally inserted central catheters compared with central venous catheters in adults: a systematic review and meta-analysis. Infect Control Hosp Epidemiol. 2013; 34(9):908-918.

19. Chopra V, Flanders SA, Saint S, et al. The Michigan Appropriateness Guide for Intravenous Catheters (MAGIC): results from a multispecialty panel using the RAND/UCLA Appropriateness Method. Ann Intern Med. 2015;163(6 suppl):S1-S40.

20. Tice AD, Rehm SJ, Dalovisio JR, et al. Practice guidelines for outpatient parenteral antimicrobial therapy. IDSA guidelines. Clin Infect Dis. 2004;38(12):1651-1672.

21. McLaws M-L, Berry G. Nonuniform risk of bloodstream infection with increasing central venous catheter-days. Infect Control Hosp Epidemiol. 2005;26(8):715-719.

22. Chopra V, Anand S, Hickner A, et al. Risk of venous thromboembolism associated with peripherally inserted central catheters: a systematic review and meta-analysis. Lancet. 2013;382(9889):311-325.

23. Chopra V, Kuhn L, Flanders SA, Saint S, Krein SL. Hospitalist experiences, practice, opinions, and knowledge regarding peripherally inserted central catheters: results of a national survey. J Hosp Med. 2013;8(11):635-638.

24. Chopra V, Govindan S, Kuhn L, et al. Do clinicians know which of their patients have central venous catheters? Ann Intern Med. 2014; 161(8):562.

25. Reilly L, Sullivan P, Ninni S, Fochesto D, Williams K, Fetherman B. Reducing foley catheter device days in an intensive care unit: using the evidence to change practice. AACN Adv Crit Care. 2006;17(3): 272-283. 